\title{
Growing optimization of suspension cultures of medicinal plant cells
}

\author{
Andrey Kolomietc, Nadezda Nicolaeva, Victoria Larina, and Nataliya Chupakhina* \\ School of Life Sciences, Immanuel Kant Baltic Federal University, 236016 Kaliningrad, Russia
}

\begin{abstract}
Suspension cell cultures allow to save plant material when obtaining biologically active compounds of natural origin. As a result of the studies, optimal parameters were selected to increase the formation of biologically active metabolites in suspension cell cultures of such medicinal plants as Maackia amurensis Rupr., Hyssopus officinalis L. and Saposhnikovia divaricata (Turcz.) Schischk. Medicinal plants are a large group of plants used as raw materials for the production of medicinal and preventive drugs for medical and animal use. The assortment of phytopreparations is constantly expanding due to the increased demand for natural remedies, due to their less aggressive and toxic nature compared to synthetic ones [1]. Cultivation of medicinal plants in the form of isolated cells in vitro is one of the most modern technologies for rapidly obtaining a large biomass of plant material with stable growth features year-round under controlled conditions [2]. It is known that cells in vitro grow faster and have peculiarities of synthesis and accumulation of biologically active substances compared to intact plants [3]. Isolated cells, unlike tissue cells, also have an advantage for their use as a source of active metabolites, since they have the ability to release these compounds into the intercellular space [4]. The goal of this paper was to select parameters for increasing the biosynthetic activity of cultured suspension cultures of medicinal plant cells in vitro by optimizing cultivation conditions and introducing precursors of secondary metabolite biosynthesis into the nutrient media.
\end{abstract}

\section{Objects and study methods}

The medicinal plants of Siberia and the Far East, collected and obtained in 2018 from the collection of the Gorno-Altaisk Botanical Garden (Russia, Republic of Altai, Shebalinskiy district, Kamlak village, Chisty Lug tract) - Maackia amurensis Rupr., Hyssopus officinalis L. and Saposhnikovia divaricata (Turcz.) Schischk.

Plant seeds were prewashed with detergent, sterilized with $75 \%$ ethanol solution and $20 \%$ sodium hypochlorite solution, then washed three times with sterile distilled water. Sterile seeds were placed in vessels filled with Murashige-Skuga (MS) nutrient medium containing 3\% sucrose and $0.7 \%$ agar-agar without growth stimulants and exposed in a thermostat at $24 \pm 1{ }^{\circ} \mathrm{C}$ until seedlings appeared, after which seedlings were transferred to light cabinet conditions with a $168^{-1} \mathrm{~h}$ photoperiod at $24 \pm 2{ }^{\circ} \mathrm{C}$ [5].

\footnotetext{
${ }^{*}$ Corresponding author: $\underline{\text { natalie-tch@yandex.ru }}$
} 
To obtain callus cultures, we used sterile 14-day-old seedlings and several versions of gelled media containing $0.7 \%$ agar-agar, differing in growth regulators. The nutrient media were sterilized by autoclaving: $20 \mathrm{~min}$ of the main mode at $121{ }^{\circ} \mathrm{C}$, with an additional pressure of 0.7 to 0.8 atmospheres.

In the first stage, calluses were grown in Petri dishes $(60 \mathrm{~mm}$ diameter $)$, then they were divided into 5 parts, and then they were re-grown under constant stirring in $100 \mathrm{ml}$ flasks (20-40 $\mathrm{ml}$ of suspension per flask) on a shaker.

The growth index (I), specific growth rate in the exponential phase $(\mu)$, economic coefficient $(\mathrm{Y})$, and doubling time $(\tau)$ were calculated according to standard methods [6].

Biomass of in vitro cell cultures was dried by lyophilization using a freeze drying Triad (Labconco, USA). Vacuum of 0.05 mbar and cooler temperature of minus $80^{\circ} \mathrm{C}$ were chosen as constant lyophilization conditions. The sample's temperature during drying was minus $20{ }^{\circ} \mathrm{C}$, and the duration of drying time was $15 \mathrm{~h}$. Lyophilically dried biomass samples were extracted with $60 \%$ methanol (biomass: extractant ratio, 1:30) under constant stirring using a thermoshaker $\left(20^{\circ} \mathrm{C}, 1400 \mathrm{rpm}\right)$ three times for $40 \mathrm{~min}$. After each interval, the extracts were centrifuged $\left(20^{\circ} \mathrm{C}, 4500 \mathrm{rpm}, 10 \mathrm{~min}\right)$. The supernatant was selected and used for qualitative and quantitative analysis of phytocomponents.

To determine the qualitative and quantitative composition of the BAS of medicinal plants, we used methods of high-performance liquid chromatography (HPLC), thin-layer chromatography (TLC), UV-spectroscopy, guided by pharmacopoeial articles [7].

All experiments were performed in at least threefold replications.

The obtained data were processed statistically.

\section{Study Results}

Suspension cultures of cells of medicinal plants of Maakia amurskaya, Saphnikova prostrate and Hyssopus medicinalis were grown on nutrient media containing per 1,000 ml: base medium - MS, sucrose - $30 \mathrm{~g}$; myo-inositol - $100 \mathrm{mg}$; 6-benzylaminopurine (BAP) $0.5 \mathrm{mg}$; s-naphthylacetic acid (NAA) - $2 \mathrm{mg}$; casein hydrolysate - $50 \mathrm{mg}$ and thiamine $0.1 \mathrm{mg}$ with addition:

- for Maackia amurensis: methyl jasmonate $-0.5 \mathrm{mg}$;

- for Saposhnikovia divaricata: Triton X-100 -2 mg;

- for Hyssopus officinalis: gibberellic acid $\left(\mathrm{HA}_{3}\right)-2 \mathrm{mg}$.

7-O-gentiobioside formonononetin (GBF), daisin, genistein, and 10-3-Ogentiobiosylmaacchia were identified as active biochemical compounds in Maakia amur cell culture. The amount of cimifugin, $\beta$-sitosterol, psoralen, and scopoletin was determined in the cell culture of Saposhnikovia divaricata. In the case of suspension cultures of hyssop, the content of hyssop, umbelliferon, diosmin, and chlorogenic acid was assessed. The total amount of these compounds for each plant illustrated the ability of the suspension cell culture to accumulate biologically active substances.

The main characteristics indicating the correctness of the choice of optimal parameters of cell culture suspension were the following: dry and crude biomass content, culture viability, and total content of biochemical compounds. Based on these parameters, we calculated indicators such as the growth index (I), the specific growth rate in the exponential phase $(\mu)$, and the economic coefficient (Y). The results of the empirical data are shown in Figures 1 and 2.

During the experiment to study the cultivation temperature effect on the main characteristics of callus culture growth, the temperature from 18 to $34{ }^{\circ} \mathrm{C}$ was used in increments of $8{ }^{\circ} \mathrm{C}$ (Figure 1a). The experiment examined the time interval from 7 to 35 days at 7-day intervals to assess the effect of cultivation duration on the same parameters (Figure 1b). 

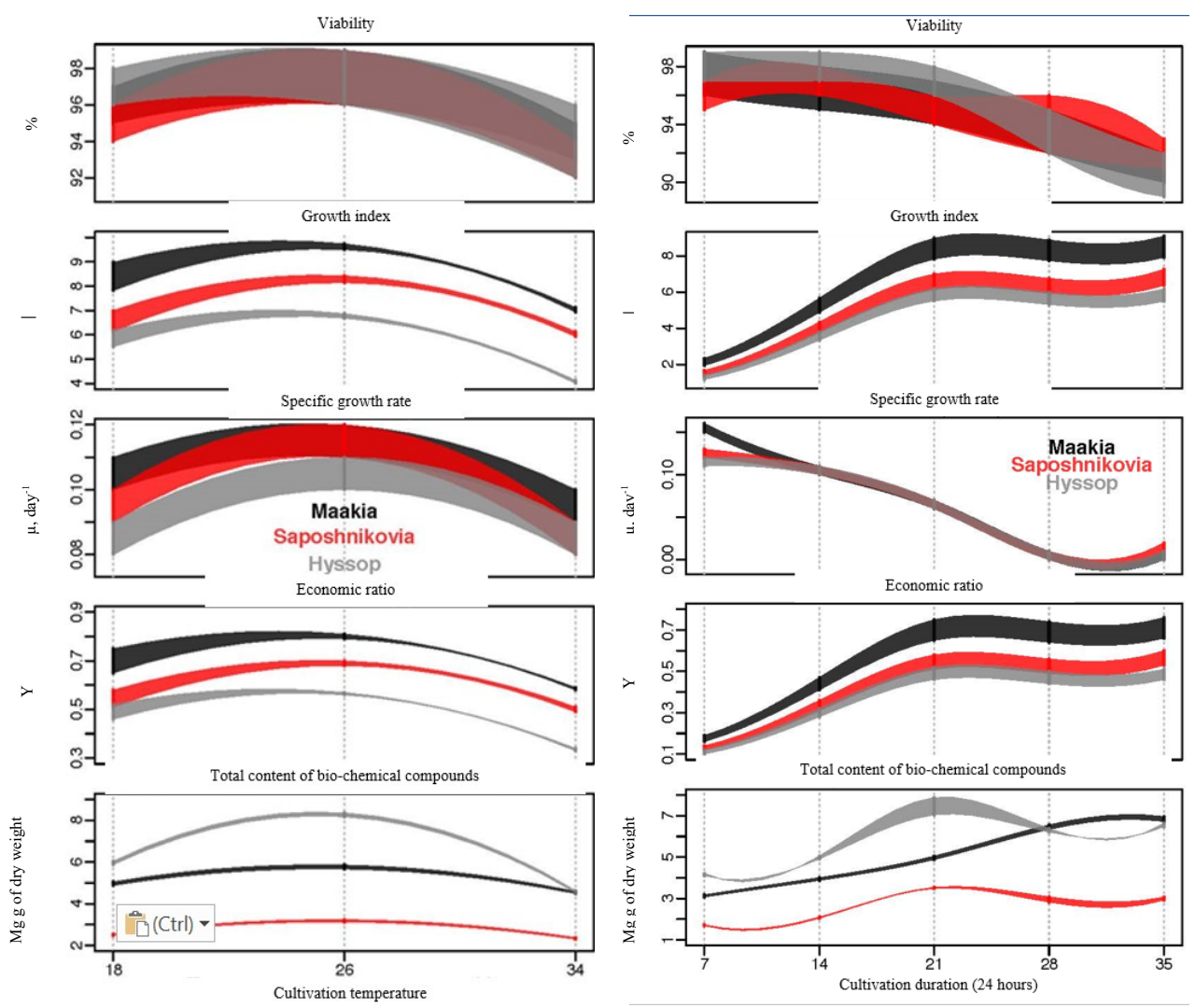

a

$\mathrm{b}$

Fig. 1. Influence of temperature (a) and cultivation duration (b) on the characteristics of suspension cell cultures

The maximum values of medicinal plant cell culture viability, biochemical content, economic coefficient, growth index and specific growth rate were observed at a temperature of $26{ }^{\circ} \mathrm{C}$ and a cultivation duration of 21 days. Also an important parameter for growing suspension cell cultures is the stirring frequency (rocker rotation speed). During the experiment, the rocker rotation speed varied from 50 to $150 \mathrm{rpm}$ (Figure 2). 

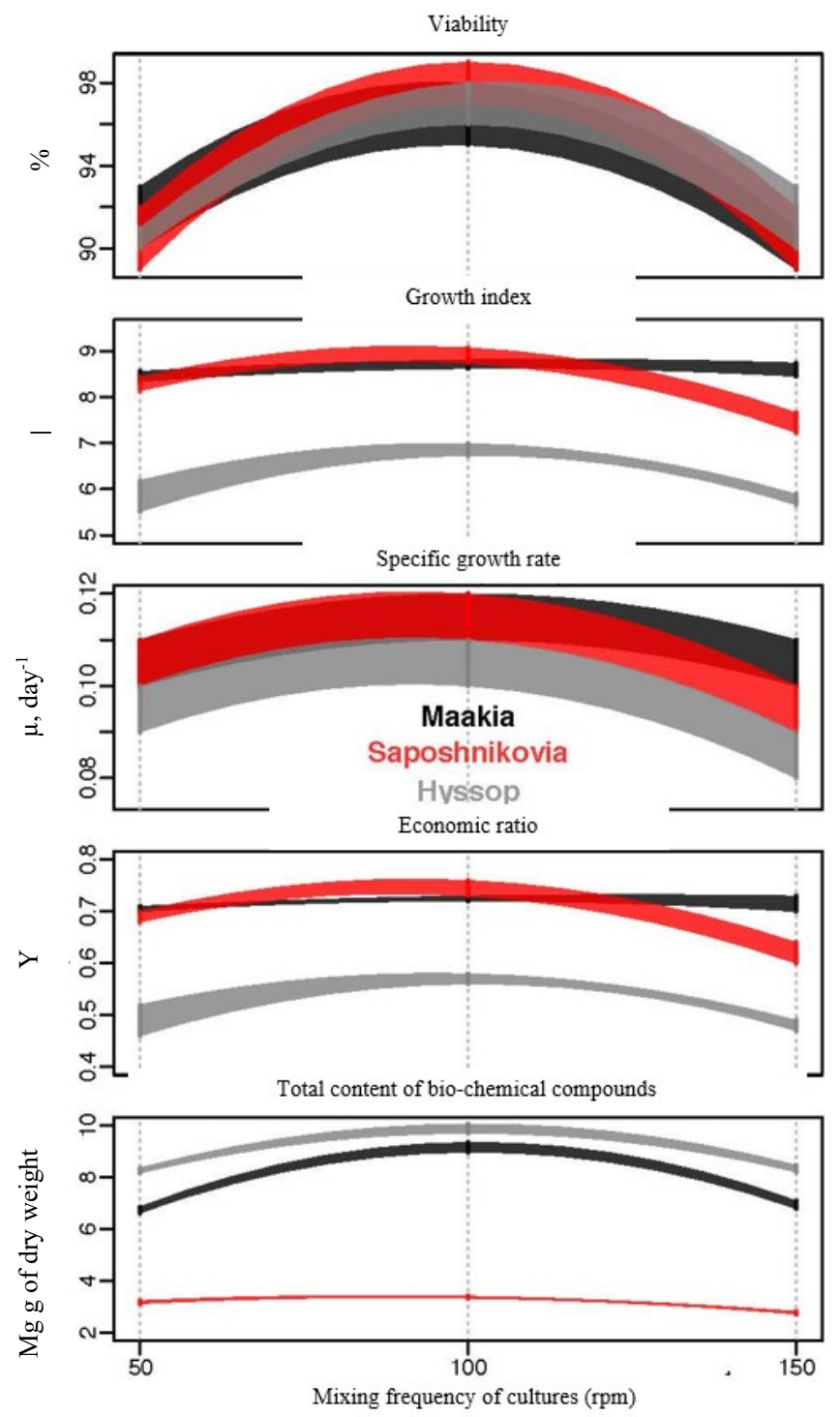

Fig. 2. Influence of culture mixing frequency on the characteristics of suspension cell cultures

The maximum values of cell culture viability and content of biochemical compounds in them were achieved at a rocker rotation speed of $100 \mathrm{rpm}$, also at this rotation speed, the highest values of the growth index and specific growth rate were noted.

Since the characteristics of plant cell cultures are affected not only by medium composition, but also by the addition of substances that actively affect growth and biochemical parameters, nutrient medium with the addition of methyl jasmonate was chosen for suspension cultures of Maakia amurica as components modifying the base medium composition [8]. Methyl jasmonate was mentioned in the literature as an effective elicitor, along with the detergent Triton X-100 [9, 10]. Therefore, Triton X-100 added medium was chosen and proved to be effective as a precursor of BAS for growing suspension cultures of Saphnikovia prostrata. Suspension cultures of hyssop demonstrated the accumulation of biologically active compounds when GAs was added to the medium as a precursor. 


\section{Conclusions}

Thus, it was found that the optimal cultivation parameters of suspension cultures of medicinal plants cells for obtaining biologically active substances of Maackia amurensis, Hyssopus officinalis and Saposhnikovia divaricata (Turcz, Hyssopus officinalis L. and Saposhnikovia divaricata (Turcz.) Schischk) were 21 days at $26{ }^{\circ} \mathrm{C}$ and grown on a shaker at $100 \mathrm{rpm}$ on media containing methyl jasmonate, GAs and Triton X-100 as precursors of active compound biosynthesis respectively.

\section{References}

1. A.M. Alrashedi, Study of morphogenetic potential of WITHANIA SOMNIFERA L. and biological activity of its exometabolites IN VITRO. Autoabstract (K.A. Timiryazev Moscow Agricultural Academy, Moscow, 2018)

2. R.G. Butenko, Biology of Higher Plant Cells in Vitro and Biotechnology Based on Them (FBC Press, Moscow, 1999)

3. K.S. Shin, J. of Microbiology, 42, 1 (2004)

4. A.A. Zagorskaya, E.V. Deineko, Plant Physiology J., 64, 6 (2017)

5. T. Murashige, F. Skoog, Physiologia plantarum J. , 15, 3 (1962)

6. E.S. Sukhanova, N.D. Chernyak, A.M. Nosov, Biotechnology J., 4 (2010)

7. Site about registration of Drugs in Russia and EAEU (CIS) https://pharmacopoeia.ru/

8. T.M. Kolomiets, V.I. Malyarovskaya, M.V. Gvasalia, L.S. Samarina, R.N. Sokolov, Agr. biol. J., 3 (2014)

9. A.A. Erst, L.N. Zibareva, E.S. Filonenko, T.V. Zheleznichenko, Chemistry of plant raw materials J., 4 (2018)

10. R. Thimmaraju, Engineering in Life Sciences J., 9 (2009) 